\title{
Prediction of Matrix Failure in Fibre Reinforced Polymer Composites
}

\author{
J. Wang ${ }^{1}$ and W. K. Chiu ${ }^{2}$ \\ ${ }^{1}$ Air Vehicles Division, Defence Science and Technology Organisation, 506 Lorimer Street, Fishermans Bend, VIC 3207, Australia \\ ${ }^{2}$ School of Mechanical Engineering, Monash University, Clayton, VIC 3800, Australia \\ Correspondence should be addressed to J. Wang; john.wang@dsto.defence.gov.au
}

Received 16 January 2013; Revised 16 June 2013; Accepted 18 June 2013

Academic Editor: Tae Jin Kang

Copyright (c) 2013 J. Wang and W. K. Chiu. This is an open access article distributed under the Creative Commons Attribution License, which permits unrestricted use, distribution, and reproduction in any medium, provided the original work is properly cited.

Recent development has enabled fibre and matrix failure in a fibre reinforced composite material to be predicted separately. Matrix yield/failure prediction is based on a Von Mises strain and first strain invariant criteria. Alternative matrix failure criteria for enhanced prediction accuracy are discussed in this paper. The proposed failure envelope formed with basic failure criteria intersects with uniaxial compression, pure shear and uniaxial tensile test data points smoothly. For failure of typical neat resin, significant improvement of prediction accuracy compared with measured material data is demonstrated. For a unit cell with a fibre and surrounding matrix with typical material properties, a FEM analysis indicates a significant improvement in prediction accuracy in the pure shear load case and a marginal improvement in the biaxial tensile load case. This paper also provided a preliminary discussion about the issues when material nonlinearity of the matrix material is involved.

\section{Introduction}

Fibre reinforced polymer matrix composites are being increasingly used for aircraft structures because of their superior structural performance (such as high strength, high stiffness, long fatigue life, and low density). Some recently developed military helicopters have nearly all-composite airframe structures (such as Eurocopter Tiger and Bell/Boeing V22).

In the conventional laminate theory widely used to predict the strength of fibre reinforced polymer composites, the laminae are treated as homogeneous orthotropic materials. Recent development has made it possible to extend the conventional laminate theory to predict separately the failure of the polymer matrix and fibres. In essence, this is through a microstructural analysis conducted on a unit cell of the composite material that contains a fibre and surrounding polymeric matrix to determine the correlation between the stress-strain states of the whole cell and its matrix and fibre components. This correlation is then used in a structural analysis to predict matrix or fibre failure. In a linear finite element method, the failure of the polymer matrix and fibres can be separately predicted during postprocessing of the results from a computation based on the conventional laminate theory, by correlating the element of stress-strain state with matrix and fibre stress-strain states. In principle, this micromechanical approach may also predict the interfacial failure between the fibre and matrix.

Significant work in the aforementioned area was reported by Gosse and his coworkers [1,2]. In their approach, it was proposed that two properties that control damage in the matrix are the first invariant of the strain tensor, $J_{1 \varepsilon}$, and the second invariant deviator, $\varepsilon_{\text {eqv }}$ :

$$
\begin{gathered}
J_{1 \varepsilon}=\varepsilon_{1}+\varepsilon_{2}+\varepsilon_{3}, \\
\varepsilon_{\text {eqv }}=\left\{0.5\left[\left(\varepsilon_{1}-\varepsilon_{2}\right)^{2}+\left(\varepsilon_{1}-\varepsilon_{3}\right)^{2}+\left(\varepsilon_{2}-\varepsilon_{3}\right)^{2}\right]\right\}^{0.5},
\end{gathered}
$$

where $\varepsilon_{1}, \varepsilon_{2}$, and $\varepsilon_{3}$ are principal strains.

These two strain variants are considered to be able to indicate matrix initial failure due to volume increase (dilational strain) and distortional strains, respectively. When either of these reaches its critical value, failure will occur. Typical matrix initial failure includes microcracking and 
TABLE 1: Typical resin material strength data [7].

\begin{tabular}{|c|c|c|c|c|c|c|c|}
\hline Resin & & Tensile (MPa) & Compression $(\mathrm{MPa})$ & Shear (MPa) & & Compression (\%) & Shear $(\%)$ \\
\hline \multirow{2}{*}{$\begin{array}{l}\text { Type } 1 \\
E=3.6 \mathrm{GPa} \lambda=0.35\end{array}$} & Yield strength & 58 & 96 & 50 & & & \\
\hline & Ultimate strength & 58 & 130 & 62 & Ultimate strain & 4.5 & 9.3 \\
\hline \multirow{2}{*}{$\begin{array}{l}\text { Type } 2 \\
E=3.9 \mathrm{GPa} \lambda=0.35\end{array}$} & Yield Strength & 50 & 100 & 36 & & & \\
\hline & Ultimate strength & 50 & 130 & 60 & Ultimate strain & 5.9 & 7.0 \\
\hline
\end{tabular}

delamination initiation. Note that (2) is essentially a Von Mises equivalent strain. In their work, the analysis so far is restricted to a linear elastic material model.

As a newly developed novel approach alternative to the conventional laminate theory, this method has great potential to be further developed, both in the areas of verification and validation. The analytical approach can be further assessed and improved. A wide range of tests need be conducted to validate the model or determine its limitations.

This paper aims to demonstrate that the accuracy of matrix failure prediction could be increased significantly by enhancing these two failure criteria. In addition, this paper will also discuss the issues when material nonlinearity of the matrix material is involved. Note that the approach provided in $[1,2]$ covers also fibre failure prediction, which is not discussed in this paper.

\section{Discussion about Polymer Failure Criteria}

In this section, the discussion is restricted to the linear elastic condition (the discussion about material nonlinearity is provided in Section 4) and four basic load cases will be considered, namely, uniaxial compression, pure in-plane shear, uniaxial tension, and biaxial tension; refer to Figure 1. The stress and strain states under the four load cases are as follows.

(i) Uniaxial compression

$$
\begin{gathered}
\sigma_{1}<0, \quad \sigma_{2}=\sigma_{3}=0, \\
\varepsilon_{1}=\frac{\sigma_{1}}{E}<0, \quad \varepsilon_{2}=\varepsilon_{3}=-\lambda \varepsilon_{1} .
\end{gathered}
$$

(ii) Pure in-plane shear

$$
\begin{gathered}
\sigma_{1}>0, \quad \sigma_{2}=-\sigma_{1}, \quad \sigma_{3}=0, \\
\varepsilon_{1}=\frac{(1+\lambda) \sigma_{1}}{E}>0, \quad \varepsilon_{2}=-\varepsilon_{1}, \quad \varepsilon_{3}=0 .
\end{gathered}
$$

(iii) Uniaxial tension

$$
\begin{gathered}
\sigma_{1}>0, \quad \sigma_{2}=\sigma_{3}=0, \\
\varepsilon_{1}=\frac{\sigma_{1}}{E}>0, \quad \varepsilon_{2}=\varepsilon_{3}=-\lambda \varepsilon_{1} .
\end{gathered}
$$

(iv) Biaxial tension

$$
\begin{gathered}
\sigma_{1}=\sigma_{2}>0, \quad \sigma_{3}=0, \\
\varepsilon_{1}=\varepsilon_{2}=\frac{(1-\lambda) \sigma_{1}}{E}, \quad \varepsilon_{3}=-2 \lambda \varepsilon_{1},
\end{gathered}
$$

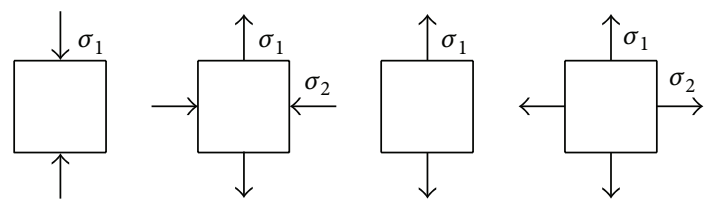

FIGURE 1: Four basic load cases considered.

where $\sigma_{1}, \sigma_{2}$, and $\sigma_{3}$ are principal stresses, $E$ is Young's modulus, and $\lambda$ is Poison's ratio.

2.1. Uniaxial Compression and Pure Shear Cases. According to $[1,2]$, the failure criterion (with the discussion restricted to the linear elastic condition, failure here just means reaching the limit of elastic range (can mean yield or other nonlinear behaviour or ultimate failure)) expressed by (2) applies to these two load cases.

It is well known that the strength of polymer materials could be affected by compressive hydrostatic stress [3-6]. For those polymer materials, to cover both uniaxial compression and pure shear load cases, a yield criterion such as the Drucker-Prager criterion would be more suitable than Von Mises criterion. (Many advanced material models are available to describe the hydrostatic effect. However, only basic failure criteria that are relatively easy to implement are considered here.) [7]

Table 1 provides yield strength data for two typical resin materials used in laminate composites.

For the prediction of yield strength, if one generates the critical value of $\varepsilon_{\text {eqv }}$ from pure shear data, the measured yield strength in the uniaxial compression case will be higher than the predicted using (2) by $11 \%$ and $60 \%$, respectively, for these two materials. This discrepancy can be removed by applying the 2-parameter Drucker-Prager criterion (based on the following):

$$
\varepsilon_{\mathrm{eqv}}+B J_{1 \varepsilon}=A
$$

where $\varepsilon_{\text {eqv }}$ and $J_{1 \varepsilon}$ are calculated using (1) and (2) with the measured failure data from compression and shear tests (Table 1); $A$ and $B$ are two parameters determined from this equation.

Failure is predicted when

$$
\varepsilon_{\mathrm{eqv}}^{\prime}=\varepsilon_{\mathrm{eqv}}+B J_{1 \varepsilon}=A,
$$

where $\varepsilon_{\text {eqv }}^{\prime}$ is the revised equivalent strain. 
Equations (9) describe the first invariant of the stress tensor, $J_{1 \sigma}$, and the second stress invariant deviator, $\sigma_{\text {eqv }}$ :

$$
\begin{gathered}
J_{1 \sigma}=\sigma_{1}+\sigma_{2}+\sigma_{3}, \\
\sigma_{\text {eqv }}=\left\{0.5\left[\left(\sigma_{1}-\sigma_{2}\right)^{2}+\left(\sigma_{1}-\sigma_{3}\right)^{2}+\left(\sigma_{2}-\sigma_{3}\right)^{2}\right]\right\}^{0.5} .
\end{gathered}
$$

Note the following relationship:

$$
\begin{gathered}
\varepsilon_{\text {eqv }}=\sigma_{\text {eqv }} \frac{1+\lambda}{E}, \\
J_{1 \varepsilon}=J_{1 \sigma} \frac{1-2 \lambda}{E} .
\end{gathered}
$$

Thus, the failure prediction equations discussed so far can all be alternatively expressed using stress variables with full equivalence.

For isotropic materials, a stress based failure criterion is also commonly used since it is often easier to use and has a clearer physical meaning (e.g., hydrostatic pressure effect shown in Drucker-Prager criterion). Alternative equations of (7) and (8) expressed in stresses are

$$
\begin{gathered}
\sigma_{\text {eqv }}+B J_{1 \sigma}=A, \\
\varepsilon_{\text {eqv }}^{\prime}=\sigma_{\text {eqv }}+B J_{1 \sigma}=A .
\end{gathered}
$$

For the materials listed in Table $1, A$ and $B$ calculated values are listed in Table 2.

2.2. Uniaxial and Biaxial Tension Cases. Before discussing the uniaxial and biaxial tension cases, we may consider an extreme case where the polymer is loaded with uniformly distributed tensile stress in all the three axial directions. It is well known that the Von Mises yield criterion is not valid in this situation. A Drucker-Prager criterion established using parameters determined from compression and shear load cases would also significantly overpredict the strength in this dilation type failure situation.

The failure pattern of typical composite matrix materials under uniaxial loading can be considered as dilation failure (rather than shear or distortional failure), as indicated from the observation that the broken sections generally are approximately perpendicular to the tensile load direction (rather than with a lager angle, i.e., shear or distortion angle), yet without significant necking.

Equation (1) adopted by Gosse and Christensen uses the volume increase of the material (summation of the 3 principal strain components) as the criterion to assess dilation type failure. Figure 2 from [1] shows the combined failure envelop formed with (1) and (2).

From Figure 2, one could see the significant singular points at the intersections between the two curves. This is an area that needs refinement (noting that a significant improvement of the Von Mises criterion over the maximum shear (Tresca) criterion is that it smooths the curve and removes the singular points in Tresca criterion).

Bardenheier [8] and Kolling et al. [9] provided experimental data from uniaxial and biaxial tension tests of three
TABle 2: Parameter $A$ and $B$ values.

\begin{tabular}{ccccc}
\hline \multirow{2}{*}{ Resin } & \multicolumn{2}{c}{ Strain-based equation } & \multicolumn{2}{c}{ Stress-based equation } \\
& $A$ & $B$ & $A$ & $B$ \\
\hline Type 1 & $32.5 \times 10^{3} \mu \varepsilon$ & 0.439 & $86.6 \mathrm{MPa}$ & 0.098 \\
Type 2 & $21.6 \times 10^{3} \mu \varepsilon$ & 1.69 & $62.4 \mathrm{MPa}$ & 0.376 \\
\hline
\end{tabular}

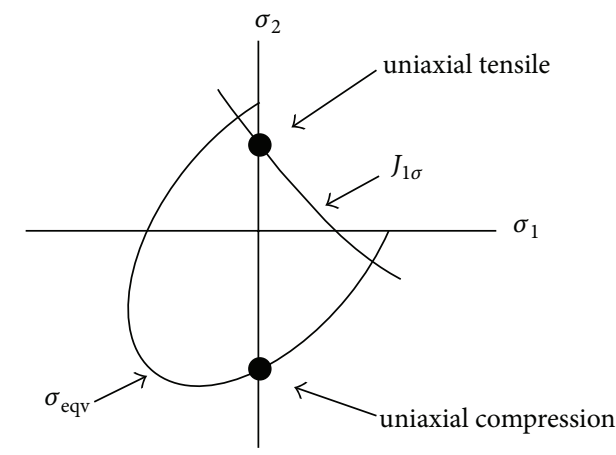

Figure 2: Failure envelope for a polymer described in [1].

types of polymer materials. Analysing these data, it is indicated that under a biaxial tensile load condition, prediction based on (1) would significantly underestimate the material strength; refer to Table 3 . The dilational failure could be more accurately predicted using the critical value of, for example with

$$
J_{1 \sigma}^{\prime}=\left({\sigma_{1}}^{2}+{\sigma_{2}}^{2}+\sigma_{3}^{2}\right)^{0.5} .
$$

This fits more closely with the experimental results. Note that since principal stresses are used, (13) is unaffected by any transformation of coordinates. As will be discussed in Section 2.3, this can join other failure criteria smoothly.

2.3. Combined Failure Envelope. A generic form of the equation that expresses the failure envelop can be as follows:

$$
F_{\varepsilon}\left(\varepsilon_{1}, \varepsilon_{2}, \varepsilon_{3}, C_{\varepsilon}\right)=0,
$$

or

$$
F_{\sigma}\left(\sigma_{1}, \sigma_{2}, \sigma_{3}, C_{\sigma}\right)=0 .
$$

Equations (14) and (15) are strain- and stress-based, respectively, where $C_{\varepsilon}$ and $C_{\sigma}$ represent a set of parameters, determined from experiment. A more generic form would be the combination of these two equations.

Equations (14) and (15) are expressed in the form of "unified" formulae applicable to all the load cases with just one formula. Alternatively, a set of equations may be used. Their combination governs the full failure envelope in the strain or stress space. No matter which forms are used, the accuracy of these failure prediction criteria must be judged by experiment results. Coupon tests are the most reliable tests, as these are conducted under the most desired load and boundary conditions in a well-controlled manner.

As mentioned earlier, though there are many advanced material models available, only basic failure criteria that are 
TABLE 3: Data from neat resin biaxial tests $[8,9]$ and errors with different prediction methods.

\begin{tabular}{lccccc}
\hline \multirow{2}{*}{ Resin } & \multicolumn{2}{c}{ Stress applied* $^{*}$} & \multicolumn{2}{c}{ Error with prediction method** (\%) } \\
& $\sigma_{1}$ & $\sigma_{2}$ & Equation $(1)$ & Equation (13) & Max stress criterion \\
\hline Type A & 0.79 & 0.79 & -36.7 & -10.5 & +21.0 \\
Type B & 0.84 & 0.50 & -25.4 & +2.3 & +16.0 \\
Type C & 0.85 & 0.80 & -39.4 & -14.3 & +15.0 \\
\hline
\end{tabular}

${ }^{*}$ Expressed using the ratio of the yield stress in the biaxial test against that in the uni-axial tensile test.

** In each method, the yield stress from the uni-axial tensile test was used to determine the critical value for prediction of yield strength in biaxial tests. A " + " sign means overprediction and a "-" sign means underprediction.

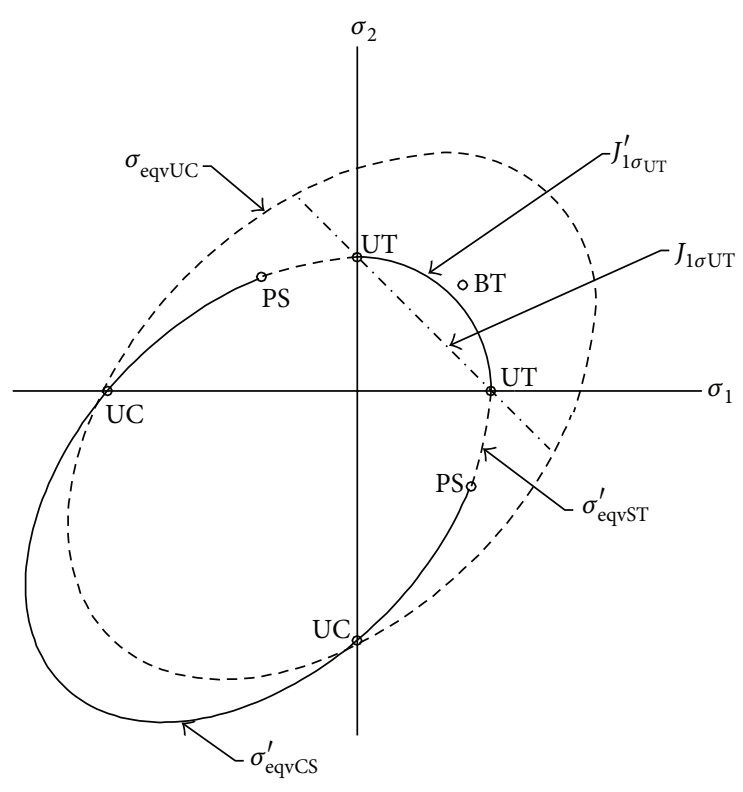

FIGURE 3: Illustration of failure envelope (plane-stress). UT = uniaxial tensile; $\mathrm{BT}=$ biaxial tensile; $\mathrm{PS}=$ pure shear; $\mathrm{UC}=$ uniaxial compression; $\sigma_{\text {eqvUC }}=\sigma_{\text {eqv }}$ with critical value determined from uniaxial compression test; $\sigma_{\text {eqvCS }}^{\prime}=\sigma_{\text {eqv }}^{\prime}$ with parameters determined from uniaxial compression and shear tests; $\sigma_{\text {eqvST }}^{\prime}=\sigma_{\text {eqv }}^{\prime}$ with parameters determined from shear and uniaxial tensile tests; $J_{1 \sigma \mathrm{UT}}=$ $J_{1 \sigma}$ with critical value determined from uniaxial tensile test; and $J_{1 \sigma \mathrm{UT}}^{\prime}=J_{1 \sigma}^{\prime}$ with critical value determined from uniaxial tensile test.

relatively easy to implement are considered here. Figure 3 illustrates the proposed failure envelope with the plane-stress condition $\left(\sigma^{3} \equiv 0\right)$. The portion of the failure envelope formed with the Drucker-Prager failure criterion has two parts. The solid line part $\left(\sigma_{\text {eqvCS}}^{\prime}\right)$ is established with the uniaxial compression and pure shear test data as discussed in Section 2.1, whilst the fine dot line part $\left(\sigma_{\text {eqvST }}^{\prime}\right)$ is established with the pure shear and uniaxial tensile data in a similar way.

This figure shows that the portion of the failure envelope formed with the Drucker-Prager failure criterion can intersect with uniaxial compression, pure shear and uniaxial tensile test data points and join with the $J_{1 \sigma}^{\prime}$ curve smoothly, that indicates significant improvement.

In the 3 -dimensional space with $\sigma_{1}, \sigma_{2}$, and $\sigma_{3}$ coordinate axes, the failure envelope is represented by two cone surfaces (formed with the Drucker-Prager failure criterion, with the axis along the line $\sigma_{1}=\sigma_{2}=\sigma_{3}$ and joined at $\sigma_{1}+\sigma_{2}+\sigma_{3}=$ 0 ) truncated by a hemisphere surface (formed with $J_{1 \sigma}^{\prime}=$ the uniaxial tensile yield stress).

\section{Lamina Unit Cell Analysis}

A unit cell analysis using a finite element method was further conducted with the four typical load cases discussed previously. The failure prediction based on (1) and (2) was compared with that based on (12) and (13) when both fibre and matrix are present. As the focus is on such a relative comparison, some factors such as the residual stress effect (due to the difference between curing and service temperatures, moisture ingression, chemical shrinkage, etc.) are not included.

Since the discussion in Section 2 indicated that the failure criteria proposed in this paper could predict neat resin failure more accurately, (12) and (13) are used as a yardstick in this comparison.

3.1. Finite Element Model. The following typical material properties are assumed:

(i) fibre Young's modulus $\left(E_{f}\right)=200 \mathrm{GPa}$,

(ii) fibre Poisson ratio $\left(\lambda_{f}\right)=0.3$,

(iii) matrix Young's modulus $\left(E_{m}\right)=3.6 \mathrm{GPa}$,

(iv) matrix Poisson ratio $\left(\lambda_{m}\right)=0.35$,

(v) Fibre volume fraction $\left(V_{f}\right)=0.5$.

Referring to Figure 4, with a relatively low fibre volume fraction of 0.5 , a "square" pattern fibre distribution in the matrix can be assumed (as opposed to a "hexagon" pattern). A unit cell was extracted with two different orientations. A FEM model is shown in Figure 5. Considering the symmetrical condition, only one-eighth of the unit cell was built.

The load condition is applied in the form of uniform surface displacement. Symmetry boundary conditions are applied to three orthogonal surfaces. The remaining unloaded surfaces were given a uniform displacement that results in the gross load on the surface being zero, to reflect the "Poisson effect." The loading and boundary conditions applied ensure all the surfaces are kept straight, as required for the simulation of a unit cell in a lamina structure. This method was benchmarked against the rule of mixtures and proved to be accurate. Further discussion about this could be found in [12].

\subsection{Results and Discussion}

3.2.1. Compression and Shear Load Cases. The unit cell shown in Figure 4(a) was considered. As expected, for the matrix material in the uniaxial compression case, high compressive 


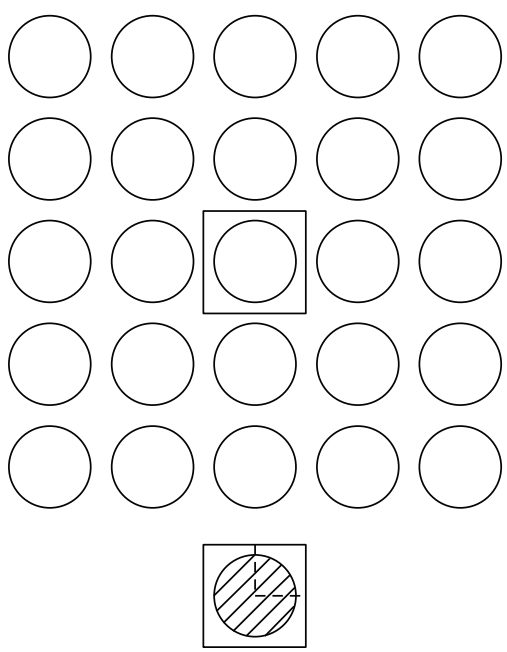

(a) Orientation 1

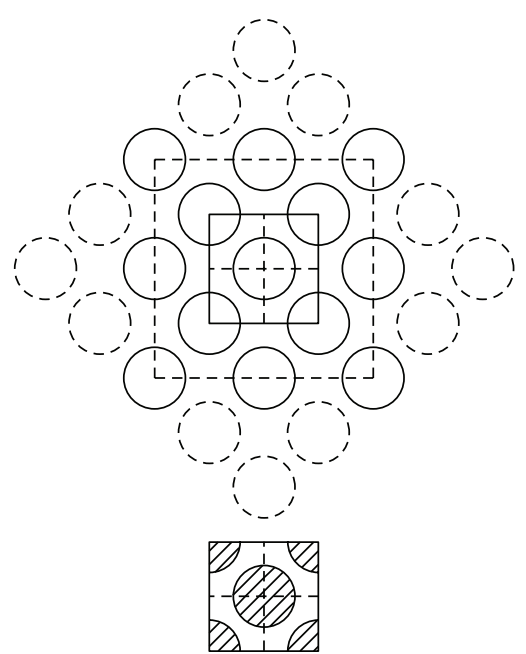

(b) Orientation 2

FIGURE 4: Unit cells with two orientations $\left(V_{f}=0.5\right)$.

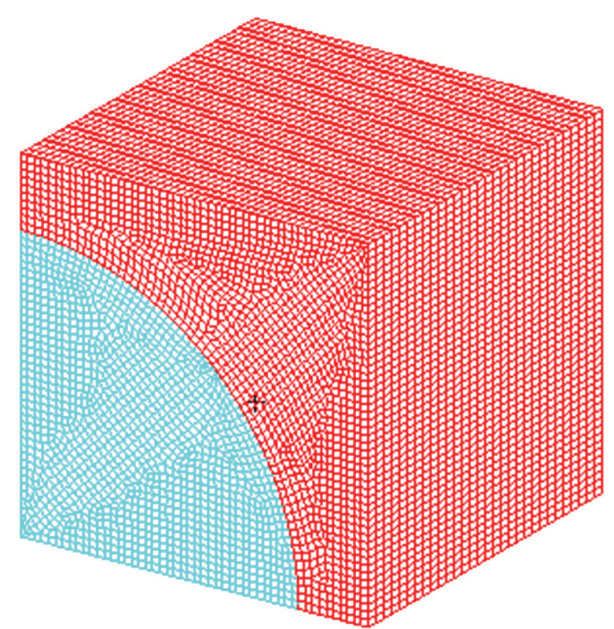

FIGURE 5: A unit cell FEM mesh (one-eighth model of the unit cell in Figure 4(a)).

stress concentration (over 4 times the average strain of the unit cell) occurred at the area indicated in Figure 6(a). Due to the Poisson effect from the stiff fibre, the other two stress components perpendicular to the applied load are also compressive in the high stress concentration area. This results in a relatively high hydrostatic stress in this area, and thus, the strength predicted with the Drucker-Prager type criterion is much higher than that with Von Mises criterion.

In contrast, in the pure shear case, high compressive and tensile stress concentration occurred separately at the areas shown in Figure 6(b). This results in the strength predicted with Drucker-Prager type criterion to be much less than that with Von Mises criterion in the tensile stress concentration area.

With a load applied that resulted in 0.01 gross strain, the calculated maximum effective stress values are listed in Table 4 .

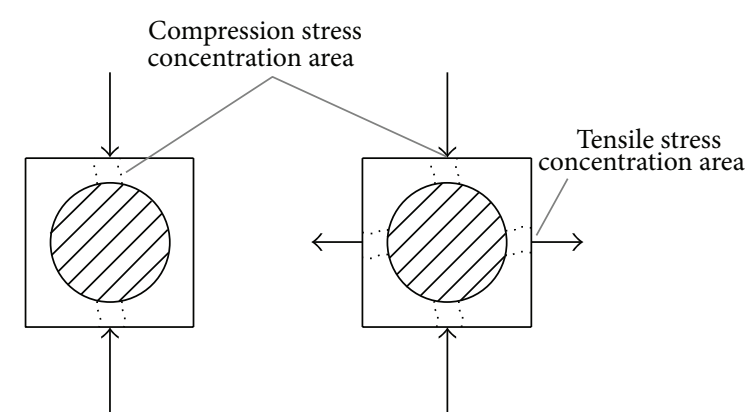

(a)

(b)

FIGURE 6: Stress concentration areas in uniaxial compression and "pure shear" load cases.

TABle 4: Predicted maximum $\varepsilon_{\text {eqv }}$ and $\sigma_{\text {eqv }}^{\prime}$ values under uni-axial compression and pure shear loadings.

\begin{tabular}{lcccc}
\hline & $\begin{array}{c}\text { (1) Uni-axial } \\
\text { compression }\end{array}$ & (2) Pure shear & Ratio (2)/(1) & Discrepancy \\
\hline$\varepsilon_{\text {eqv }}$ & 0.0574 & 0.0624 & 1.09 & $60.6 \%$ \\
$\sigma_{\text {eqv }}^{\prime}$ & $1.14 E 2 \mathrm{MPa}$ & $2.00 E 2 \mathrm{MPa}$ & 1.75 & \\
\hline
\end{tabular}

For practical applications of the approach described in [1, 2], the failure strength of the matrix is generally determined using laminate specimen tests rather than from neat resin tests (the effect of scale difference between the unit cell and lamina structure is generally not considered in this approach, refer to [1], whilst this calibration may, to a certain degree, take the scale effect into consideration). If the strength is calibrated using the uniaxial compression strength for material type 1 (Table 1), when predicting strength in the pure shear load case, as indicated in Table 4 , the difference between the original approach and the proposed revised approach is $60.6 \%$. 
TABLe 5: Predicted maximum $J_{1 \varepsilon}$ and $J_{1 \sigma}^{\prime}$ values under uni-axial and biaxial tension loadings—orientation 1 .

\begin{tabular}{lcccc}
\hline & $\begin{array}{c}\text { (1) Uni-axial } \\
\text { tension }\end{array}$ & (2) Biaxial tension & Ratio (2)/(1) & Discrepancy \\
\hline$J_{1 \varepsilon}$ & 0.0376 & 0.0438 & 1.18 & $3.4 \%$ \\
$J_{1 \sigma}^{\prime}$ & $2.73 E 2 \mathrm{MPa}$ & $3.12 E 2 \mathrm{MPa}$ & 1.14 & \\
\hline
\end{tabular}

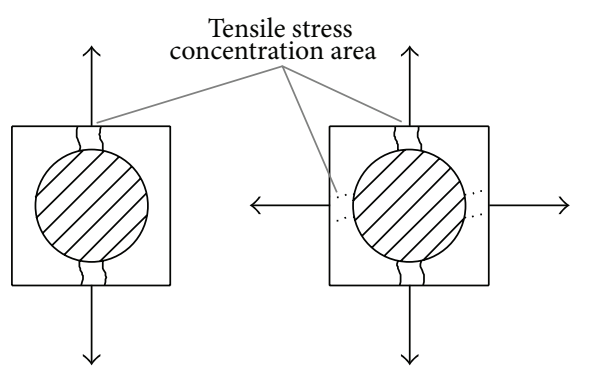

(a)

(b)

FIGURE 7: Stress concentration areas in uniaxial and biaxial tension load cases-orientation 1 .

\subsubsection{Uniaxial and Biaxial Tension Load Cases. Figure 7} shows the stress concentration areas in these two load cases for the unit cell shown in Figure 4(a). In the biaxial tension case, each of the two stress concentration areas was affected mainly by one of the two loads. Thus, there is no significant biaxial tensile stress. Table 5 lists maximum $J_{1 \varepsilon}$ and $J_{1 \sigma}^{\prime}$ values. Consequently, when using the two different methods calibrated using uniaxial tensile state data to predict biaxial state strength, the difference is only $3.4 \%$.

The unit cell shown in Figure 4(b) was subsequently used in the comparison. The maximum critical value locations in the uniaxial tensile load case are at " $a$ " positions shown in Figure 8(a), whilst the maximum critical value locations in the biaxial tensile load case are at " $b$ " positions shown in Figure 8(b). These maximum critical values are listed in Table 6.

As shown in Table 6, when using the two different methods calibrated using uniaxial tensile state data to predict biaxial state strength, the difference is $6.1 \%$, in terms of the maximum critical values in the whole matrix; however, for a specific critical location such as at " $b$ " locations, the two methods yield a significant difference. This might have a complicated effect on determining the correlation between the element stress-strain and matrix and fibre stress-strain states.

Note that when a further tensile load is applied along the fibre direction, resulting in a more uniformed stress state among the three principal stresses at the critical location, a higher difference was predicted between these two criteria. Since this is beyond the four load cases considered, detailed description is not presented.

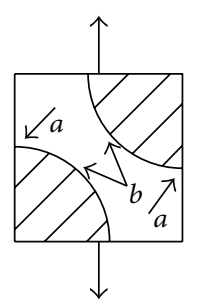

(a)

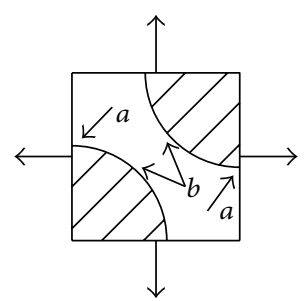

(b)
FIGURE 8: Stress concentration areas in uniaxial and biaxial tension load cases-orientation 2.

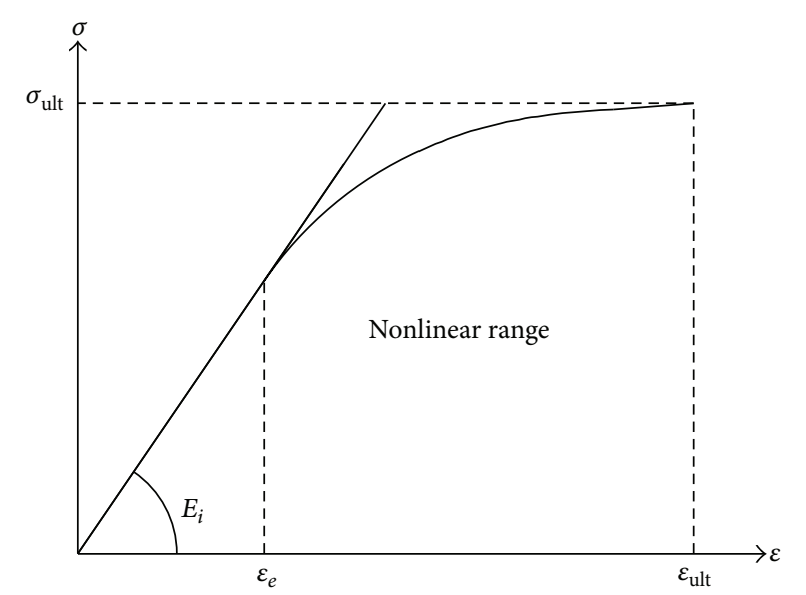

FIGURE 9: Nonlinear stress-strain relationship. $E_{i}=$ initial or nominal modulus, $\varepsilon_{e}=$ elastic limit strain, $\varepsilon_{\text {ult }}=$ ultimate strain, and $\sigma_{\text {ult }}=$ ultimate stress.

\section{Preliminary Discussion about Material Nonlinearity}

Once the matrix stress-strain state is within the nonlinear range, its modulus would no longer be constant $[7,13,14]$. Thus, in the microstructural analysis, as shown in Figure 9, the correlation between the stress-strain states of the whole unit cell and its matrix and fibre components becomes stress dependent (or strain dependent). As a strain hardening material the effective modulus of the matrix could be significantly lower than the nominal (initial) modulus.

In principle, a nonlinear structural analysis can be conducted using a finite element method with laminar nonlinear modulus properties based on the conventional laminate theory. The failure of the polymer matrix and fibres can be separately predicted by correlating the element stressstrain state with matrix and fibre stress-strain states at each iteration step. However, this could become impractical due to the computational expense of the nonlinear microstructural analysis at every iteration step.

4.1. Matrix Dominant Case. Where the effect of material nonlinearity on laminate strength prediction is most apparent would be a strong matrix dominant case, such as to predict 
TABLE 6: Predicted $J_{1 \varepsilon}$ and $J_{1 \sigma}^{\prime}$ values at critical points under uni-axial and biaxial tension loadings—orientation 2.

\begin{tabular}{|c|c|c|c|c|c|}
\hline & & (1) Uni-axial tension & (2) Biaxial tension & Ratio $(2) /(1)$ & Difference \\
\hline \multirow{2}{*}{ Point $a$} & $J_{1 \varepsilon}$ & 0.0141 & 0.0334 & 2.37 & \multirow{2}{*}{$1.0 \%$} \\
\hline & $J_{1 \sigma}^{\prime}$ & $1.05 E 2 \mathrm{MPa}$ & $2.44 E 2 \mathrm{MPa}$ & 2.32 & \\
\hline \multirow{2}{*}{ Point $b$} & $J_{1 \varepsilon}$ & 0.00978 & 0.0435 & 4.44 & \multirow{2}{*}{$49.0 \%$} \\
\hline & $J_{1 \sigma}^{\prime}$ & $1.02 E 2 \mathrm{MPa}$ & $3.05 E 2 \mathrm{MPa}$ & 2.98 & \\
\hline \multirow{2}{*}{ Max } & $J_{1 \varepsilon}$ & 0.0141 & 0.0435 & 3.09 & \multirow{2}{*}{$6.1 \%$} \\
\hline & $J_{1 \sigma}^{\prime}$ & $1.05 E 2 \mathrm{MPa}$ & $3.05 E 2 \mathrm{MPa}$ & 2.90 & \\
\hline
\end{tabular}

TABLE 7: Mechanical properties of AS4/3501-6 lamina-source [10].

\begin{tabular}{lccc}
\hline \multicolumn{1}{c}{ Elastic constants } & \multicolumn{2}{c}{ Strength properties } \\
\hline Longitudinal modulus, $E_{11} \mathrm{GPa}$ & 142 & Longitudinal tensile, $\left[\sigma_{11 t}\right] \mathrm{MPa}$ & 2280 \\
Transverse modulus, $E_{22} \mathrm{GPa}$ & 10.3 & Longitudinal compressive, $\left[\sigma_{11 c}\right] \mathrm{MPa}$ & $-1769^{*}$ \\
Shear modulus, $G_{12} \mathrm{GPa}$ & 7.2 & Transverse tensile, $\left[\sigma_{22 t}\right] \mathrm{MPa}$ & 57 \\
Poisson's ratio, $v_{12}$ & 0.27 & Transverse compressive, $\left[\sigma_{22 c}\right] \mathrm{MPa}$ & -228 \\
& & Shear, $\left[\tau_{12}\right] \mathrm{MPa}$ & 71 \\
\hline
\end{tabular}

${ }^{*}$ Value adjusted according to microbuckling consideration [11].

delamination on-set load for a laminate subject to a throughthickness shear load. The material nonlinearity is reflected directly in the load-displacement relationship of the laminate and thus needs to be taken into account for an accurate microstructure analysis and accurate prediction for the load limit of the laminate.

It would be feasible to conduct a global analysis run with the conventional laminate theory model (may include material nonlinearity) and give an initial assessment to matrix failure based on the "standard" linear microstructural analysis approach to locate the critical area and the local load condition. Then conduct a local model FEM structural analysis in conjunction with a unit cell microstructural analysis, in which the nonlinear material property is considered. The computational expense might be manageable.

4.2. Fibre Dominant Case. We may examine a typical fibre dominant load case, a panel made of AS4/3501-6 prepreg tape with a quasi-isotropic layup [0/-45/45/90]s, under a uniaxial compression in-plane load along the 0 -direction.

The material properties are listed in Table 7. The measured nonlinear stress-strain curve for the angle layers is provided in Figure 10. If we apply a linear FEM analysis based on the conventional laminate theory with the linear property data from Table 7 , the computation predicts that the angle ply will fail first and the laminate strength will be significantly lower than that from the nonlinear analysis or the measured experimental result by $35 \%$ [11].

An alternative way of linear approximation is to consider that the laminate failure occurs when the 0-ply ultimate stress is reached, when the angle ply has not yet reached its ultimate strain (according to Figure 10). The elastic shear modulus value shown in Table 7 is used in the calculation for the angle layer up to the laminate failure (linear approximation). This will overpredict the strength by around 5\% (nonconservative).

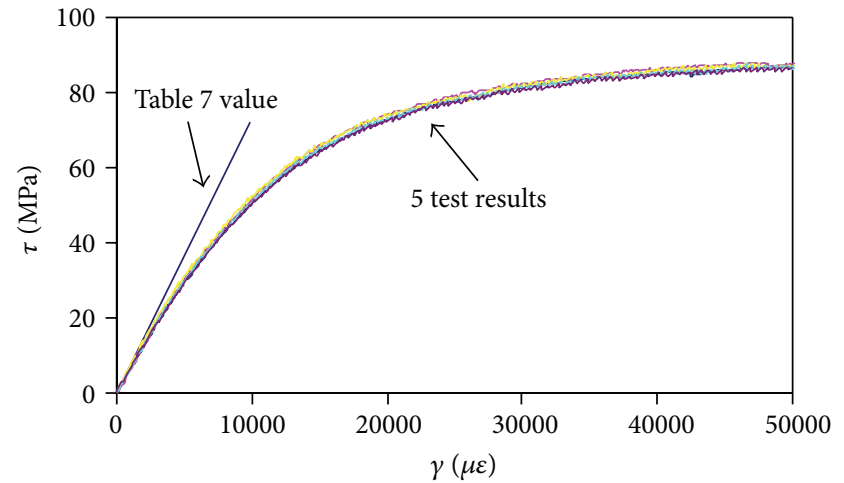

FIGURE 10: Shear stress-strain curve of AS4/3501-6 $\left( \pm 45^{\circ}\right.$ tension test) [11].

Though arguably $5 \%$ overprediction may not be negligible, the aforementioned does indicate that, with a linear approximation, when the ultimate strain of a matrix dominant lamina stress-strain component is used, the predicted loading capacity is much closer to the nonlinear analysis than that when the ultimate stress is used (35\% under prediction). This is due to the matrix strain hardening behaviour and the insignificant contribution of the matrix dominated stress component in the overall loading capacity of the laminar.

The previous discussion relates two different ways of linear approximation. Extending this discussion, one may conclude that in the microstructural analysis (postprocession of a FEM linear approximation analysis) to use the ultimate strain of a matrix would result in a nonconservative but much smaller strength prediction error than to use the ultimate stress of the matrix (conservative, much larger strength prediction error).

Thus, when a prediction based on linear analysis approximation is used in the case where the matrix material nonlinearity effect is significant, it is important to determine 


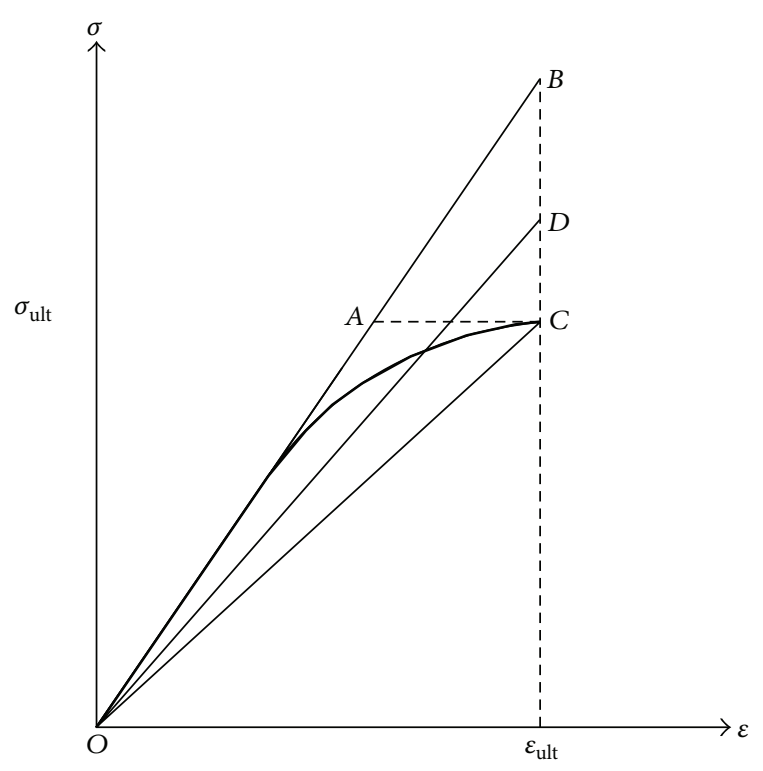

FIGURE 11: Different ways of linear approximation for nonlinear stress-strain relationship of polymer matrix or matrix dominant lamina property.

the maximum possible error and if it is acceptable in terms of the requirement/purpose of the prediction.

A further point to make is the different way to make the linearization. As shown in Figure 11, line OA uses the initial modulus in conjunction with ultimate stress as failure indication (conservative), line $\mathrm{OB}$ uses the initial modulus and ultimate strain (nonconservative), line $\mathrm{OC}$ uses a secant modulus and ultimate stress (conservative), and line OD uses the strain energy to define modulus and uses ultimate strain as failure indication. Line OD may be a good way for the linearization in terms of strength prediction error.

\section{Summary}

Recent development has enabled fibre and matrix failure in a fibre reinforced composite material to be predicted separately. Matrix yield/failure prediction is based on a Von Mises strain and first strain invariant criteria. Improvement of the matrix failure criteria for enhanced prediction accuracy is discussed and demonstrated in this paper.

For two typical resin materials considered, Von Mises yield criterion is unable to fit both yield strength values from uniaxial compression and pure shear tests. When calibrated using the measured shear yield strength, the discrepancy between the predicted and measured uniaxial compression strengths is found to be $11 \%$ and $60 \%$, respectively, whilst using a Drucker-Prager criterion, these discrepancies could be removed.

Use of the critical value of the first invariant strain, when calibrated using the uniaxial tensile yield strength, underpredicts the biaxial tensile strength significantly by over $30 \%$ on average, compared with available measurement data of neat resin materials. A revised criterion proposed in this paper could reduce the discrepancy to less than $10 \%$. The proposed failure envelope intersects with all the uniaxial compression, pure shear and uniaxial tensile test data points, and a revised tensile failure criterion. The areas governed by these criteria join each other smoothly.

For a unit cell with a fibre and surrounding matrix from a lamina with 50\% fibre volume fraction and typical material properties, a FEM analysis conducted in this study indicates that the difference between the yield strength of the matrix material predicted using Von Mises and Drucker-Prager type criteria is over $60 \%$ in the pure shear load case, when the critical values of these yield criteria are determined in the uniaxial compression load case.

The FEM analysis showed that the difference between the yield strength of the matrix material in a unit cell predicted using the first strain variant and the revised criterion reaches $6.1 \%$ in the biaxial tensile load case, when the critical values of these yield criteria are determined in the uniaxial tensile load case. With a tensile load added along the fibre direction, this difference is further increased.

This paper also provided a preliminary discussion about the issues when matrix material nonlinearity is involved.

As a newly developed novel approach alternative to the conventional laminate theory, this method has great potential to be further developed, both in the areas of verification and validation. The analytical approach can be further assessed and improved. A wide range of tests need be conducted to validated the model or determine its limitations.

\section{Acknowledgment}

The authors would like to acknowledge that this paper is the outcome of a research project of the Cooperative Research Centre for Advanced Composite Structures (CRC-ACS).

\section{References}

[1] J. H. Gosse and S. Christensen, "Strain invariant failure criteria for polymers in composite materials," in Proceedings of the 42nd AIAA/ASME/ASCE/AHS/ASC Structures, Structural Dynamics, and Materials Conference and Exhibit Technical Papers, pp. 4555, Seattle, Wash, USA, April 2001.

[2] D. L. Buchanan, J. H. Gosse, J. A. Wollschlager, A. Ritchey, and R. Byron Pipes, "Micromechanical enhancement of the macroscopic strain state for advanced composite materials," Composites Science and Technology, vol. 69, no. 11-12, pp. 19741978, 2009.

[3] S. S. Sternstain and F. A. Myers, "Yielding of glassy polymers in the second quadrant of principal stress space," Journal of Macromolecular Science B, vol. 8, pp. 537-571, 1973.

[4] P. B. Bowden and J. A. Jukes, "The plastic flow of isotropic polymers," Journal of Materials Science, vol. 7, no. 1, pp. 52-63, 1972.

[5] T. Wang, K. Kikno, and N. Mitsuo, "Effect of triaxial stress constraint on the deformation and fracture of polymers," Acta Mechanica Sinica/Lixue Xuebao, vol. 18, no. 5, pp. 480-493, 2002.

[6] Y. P. Fei, Mechanics of Fibre Reinforced Composite, Tongji University Press, Shanghai, China, 1981. 
[7] D. C. Drucker and W. Prager, "Solid mechanics and plastic analysis for limit design," Quarterly of Applied Mathematics, vol. 10, no. 2, pp. 157-165, 1952.

[8] Bardenheier, Mechanicaches Versagen von Polymerwerkstooen, Hanser, 1982.

[9] S. Kolling, A. Haufe, M. Feucht, and P. A. Du Bois, "A constitutive formulation for polymers subjected to high strain rates," in Proceedings of the 9th International LS-Dyna Users Conference, Detroit, Mich, USA, June 2006.

[10] P. K. Mallick, Composites Engineering Handbook, 1997.

[11] J. Wang, P. J. Callus, and M. K. Bannister, "Experimental and numerical investigation of the tension and compression strength of un-notched and notched quasi-isotropic laminates," Composite Structures, vol. 64, no. 3-4, pp. 297-306, 2004.

[12] J. Wang, D. Kelly, and W. Hillier, "Finite element analysis of temperature induced stresses and deformations of polymer composite components," Journal of Composite Materials, vol. 34, no. 17, pp. 1456-1471, 2000.

[13] R. K. Goldberg, G. D. Roberts, and A. Gilat, "Analytical modeling of the high strain rate deformation of polymer matrix composites," in Proceedings of the 44th AIAA/ASME/ASCE/AHS/ ASC Structures, Structural Dynamics, and Materials Conference, pp. 3264-3272, April 2003.

[14] R. K. Goldberg, Implementation of Fiber Substructuring into Strain Rate Dependent Micromechanics Analysis of Polymer Matrix Composites, National Aeronautics and Space Administration. Glenn Research Center, 2001, NASA/TMm2001-210822. 

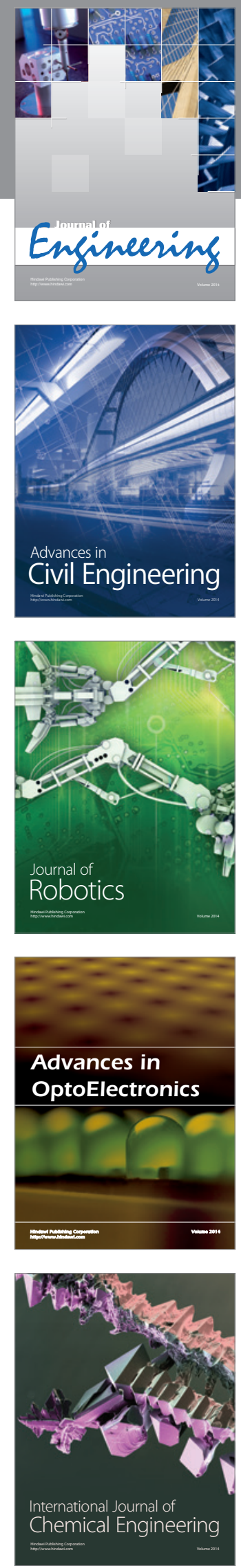

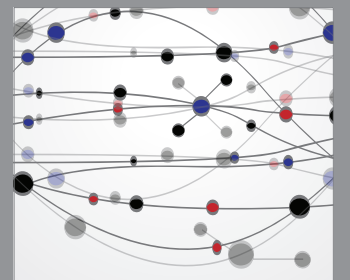

The Scientific World Journal
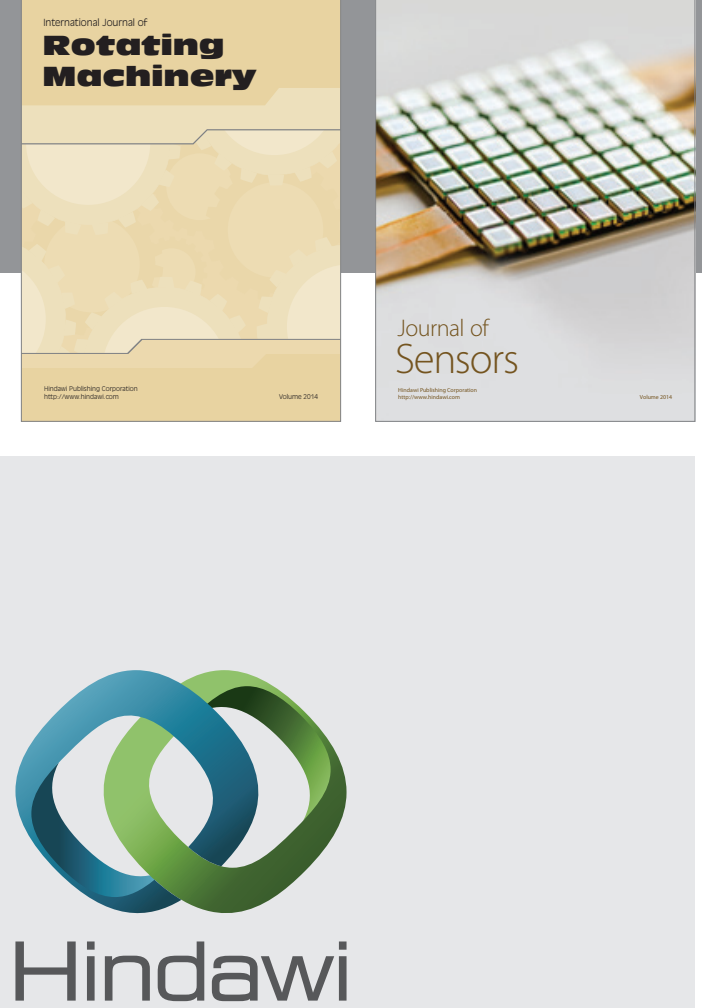

Submit your manuscripts at http://www.hindawi.com
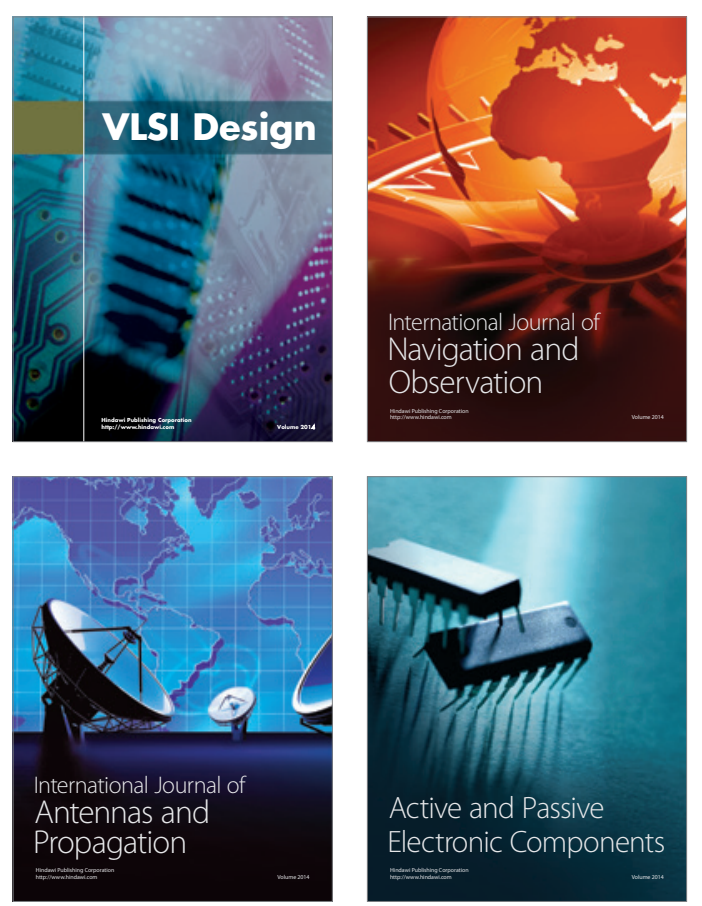
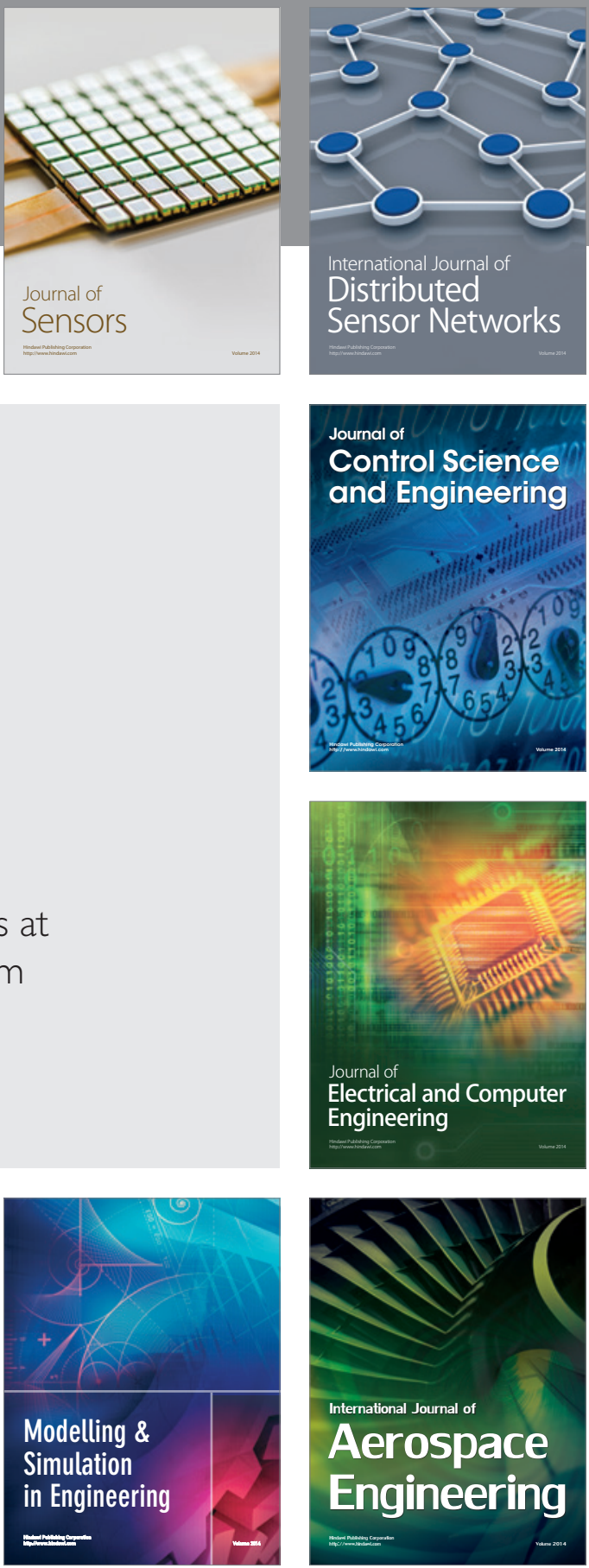

Journal of

Control Science

and Engineering
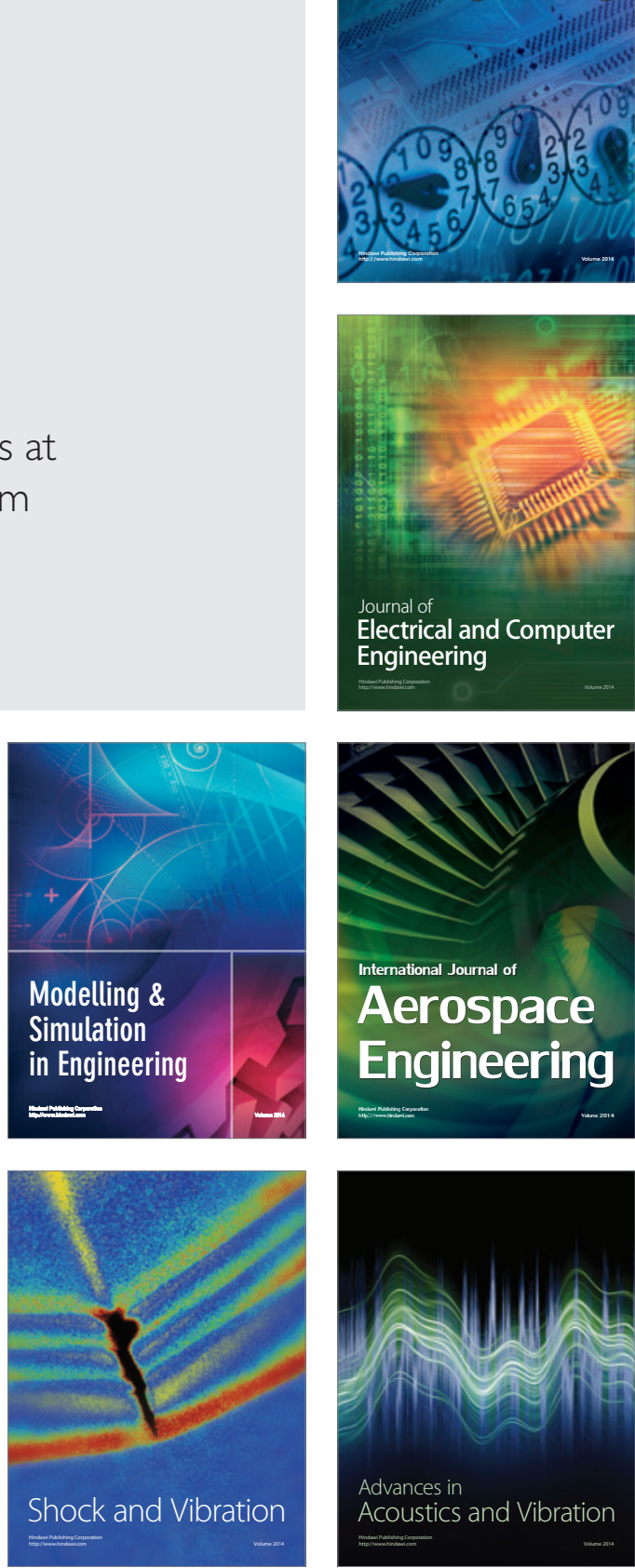